\title{
The Stroke Hyperglycemia Insulin Network Effort (SHINE) trial protocol: a randomized, blinded, efficacy trial of standard vs. intensive hyperglycemia management in acute stroke
}

\author{
Askiel Bruno ${ }^{1 *^{\dagger}}$, Valerie L. Durkalski ${ }^{2 \dagger}$, Christiana E. Hall ${ }^{3}$, Rattan Juneja ${ }^{4}$, William G. Barsan ${ }^{5}$, \\ Scott Janis ${ }^{6}$, William J. Meurer ${ }^{5}$, Amy Fansler ${ }^{7}$, and Karen C. Johnston ${ }^{7}$ \\ on behalf of the SHINE investigators
}

\begin{abstract}
Rationale Patients with acute ischemic stroke and hyperglycemia have worse outcomes than those without hyperglycemia. Intensive glucose control during acute stroke is feasible and can be accomplished safely but has not been fully assessed for efficacy.

Aims The Stroke Hyperglycemia Insulin Network Effort trial aims to determine the safety and efficacy of standard vs. intensive glucose control with insulin in hyperglycemic acute ischemic stroke patients.

Design This is a randomized, blinded, multicenter, phase III trial of approximately 1400 hyperglycemic patients who receive either standard sliding scale subcutaneous insulin (blood glucose range $\mathbf{8 0 - 1 7 9 ~} \mathrm{mg} / \mathrm{dL}$, 4.44-9.93 mmol/L) or continuous intravenous insulin (target blood glucose $80-130 \mathrm{mg} /$ $\mathrm{dL}, 4.44-7.21 \mathrm{mmol} / \mathrm{L}$ ) for up to $72 \mathrm{~h}$, starting within $12 \mathrm{~h}$ of stroke symptom onset. The acute treatment phase is single blind (for the patients), but the final outcome assessment is double blind. The study is powered to detect a $7 \%$ absolute difference in favorable outcome at 90 days.

Study outcomes The primary outcome is a baseline severity adjusted 90-day modified Rankin Scale score, defined as 0, 0-1, or $0-2$, if the baseline National Institutes of Health Stroke Scale score is $3-7,8-14$, or $15-22$, respectively. The primary safety outcome is the rate of severe hypoglycemia $(<40 \mathrm{mg} / \mathrm{dL}$, $<2.22 \mathrm{mmol} / \mathrm{L}$ ).

Discussion This trial will provide important novel information about preferred management of acute ischemic stroke patients with hyperglycemia. It will determine the potential benefits and risks of intensive glucose control during acute stroke.
\end{abstract}

\footnotetext{
Correspondence: Askiel Bruno*, Department of Neurology, Medical

College of Georgia, BI 3076, Augusta, GA 30912, USA.

E-mail: abruno@georgiahealth.edu

${ }^{1}$ Department of Neurology, Medical College of Georgia, Augusta, GA, USA

${ }^{2}$ Department of Public Health Sciences, Medical University of South Carolina, Charleston, SC, USA

${ }^{3}$ Department of Neurology and Neurotherapeutics, University of Texas Southwestern Medical Center, Dallas, TX, USA

${ }^{4}$ Division of Endocrinology, Department of Medicine, Indiana

University School of Medicine, Indianapolis, IN, USA

${ }^{5}$ Department of Emergency Medicine, University of Michigan, Ann Arbor, MI, USA

${ }^{6}$ National Institute of Neurological Disorders and Stroke, National Institutes of Health, Bethesda, MD, USA

${ }^{7}$ Department of Neurology, University of Virginia School of Medicine, Charlottesville, VA, USA
}

${ }^{\dagger}$ The amount contributed by these authors is indistinguishable

Conflict of interest: Dr Rattan Juneja receives royalties from commercial sales of the GlucoStabilizer ${ }^{\circledR}$ tool.

DOI: $10.1111 /$ ijs.12045
Key words: acute ischemic stroke, cerebral infarction, clinical trial, hyperglycemia, protocols

\section{Introduction and rationale}

Hyperglycemia is seen in approximately $40 \%$ of acute ischemic stroke patients $(1,2)$ and is associated with worse clinical outcomes (1-3). Preclinical and clinical data suggest a potential clinical benefit of intensive glucose control in the setting of acute cerebral ischemia. However, hypoglycemia, especially severe or prolonged hypoglycemia, is of greatest concern with insulin therapy (4-7). A protocol minimizing severe and prolonged hypoglycemia while controlling hyperglycemia has the potential to improve outcomes in acute stroke patients. Intensive glucose control with intravenous (IV) insulin therapy has been found to improve clinical outcomes in some nonstroke acute illnesses $(8,9)$. However, there remains clinical equipoise about how best to treat hyperglycemia during acute ischemic stroke. Results from the National Institutes of Health (NIH)-National Institute of Neurological Disorders and Stroke (NINDS) funded Treatment of Hyperglycemia in Ischemic Stroke (10) and the Glucose Regulation in Acute Stroke Patients (11) trials demonstrated safety and feasibility of insulin infusion therapy for intensive glucose control in acute ischemic stroke patients. No previous trial has fully assessed the efficacy of intensive glucose control, and current stroke guidelines therefore emphasize the need for definitive clinical trials to determine the optimal management of hyperglycemia in acute stroke (12).

As improved glucose control protocols decreased the risk of hypoglycemia, determining the efficacy and safety of intensive vs. standard glucose control in acute ischemic stroke patients became a priority. Numerous previous glucose control trials informed the Stroke Hyperglycemia Insulin Network Effort (SHINE) trial design (10,11,13-15). The SHINE trial was designed to address the key questions about hyperglycemia management in acute stroke. The primary aim of the SHINE trial is to determine the efficacy of intensive vs. standard glucose control. The secondary aim is to assess safety and therefore determine an overall risk/ benefit profile for the intensive insulin intervention vs. the standard treatment. The results of this trial will likely clarify the preferred treatment and guide clinical decision making.

\section{Design}

Stroke Hyperglycemia Insulin Network Effort is a randomized, blinded, multicenter, controlled, phase III trial of continuous 
intravenous insulin vs. standard subcutaneous (SQ) insulin in acute ischemic stroke with hyperglycemia. Additional treatments constituting usual care are allowed, including IV tissue plasminogen activator (tPA), intra-arterial tPA, and United States Food and Drug Administration (FDA) cleared endovascular devices. Throughout the study period, current American Heart Association guideline for the early management of adults with ischemic stroke will be followed (12).

Enrollment must be within $12 \mathrm{~h}$ of stroke symptom onset. Also, to maximize early treatment, enrollment should be within three-hours of arrival to the Emergency Department. The 12-h window for enrollment is based on a combination of supporting animal model data, feasibility, and generalizability considerations.

The trial is single blind during treatment and double blind for the functional outcome assessment. Blinding the treating team during treatment was felt to impose excessive risk, and having an unblinded investigator manage the study treatment for $72 \mathrm{~h}$ on an hourly basis is not feasible.

At six-weeks, serious adverse events and early follow-up information are assessed by telephone. At three-months, the primary and secondary outcomes are assessed in person. Subjects unable to return in person at three-months are assessed by telephone. The sliding dichotomy that assesses the primary outcome allows individual patients to have favorable outcomes according to their initial stroke severity, based on the National Institutes of Health Stroke Scale (NIHSS) (16). This design maximizes the generalizability of the results by enrolling a broad range of stroke severities and increases the use of individual patient data that are often lost with single dichotomized outcome definitions. The sliding dichotomy has been successfully used in a previous stroke trial (17) and is described under primary outcome.
Recruitment is aimed at capturing a broad patient population from across the United States with expected suitable representation of women and minorities. Only adults aged 18 years or older are included. To optimize recruitment, the SHINE research team includes a recruitment Principal Investigator (PI) (C. E. H.) and a NINDS recruitment specialist whose focus is to maximize timely recruitment across all sites. This team has developed individualized screening and recruitment goals and a monitoring plan for each site. The recruitment plan is designed to promptly and accurately estimate the number of sites required to complete recruitment on schedule.

The limitations of this design include potential bias due to the single-blind acute treatment period. The double-blind primary outcome assessment is designed to eliminate this bias. Potential measured and unmeasured confounders include all treatments and care outside the study treatment protocol through the 90-day outcome assessment. The secondary analysis will attempt to adjust for prespecified confounds.

\section{Patient population}

The primary target population for the SHINE trial is acute ischemic stroke patients likely to have persistent hyperglycemia during hospitalization and who can be enrolled within $12 \mathrm{~h}$ of symptom onset (Table 1). Patients receiving standard IV tPA are stratified at randomization to prevent confounding of treatment effect.

Previous data indicate that patients with history of type 2 diabetes or those with baseline blood glucose at or above $150 \mathrm{mg} / \mathrm{dL}$ $(8.32 \mathrm{mmol} / \mathrm{L})$ (even without diabetes) are likely to have persistent hyperglycemia, unless treated with insulin $(10,11,13,18)$. Patients who do not meet these criteria typically have self-limited

\section{Table 1 Eligibility criteria}

Inclusion

1. Age 18 years or older.

2. Clinical diagnosis of ischemic stroke. Neuroimaging must be done to exclude intracranial haemorrhage.

3. Protocol treatment should begin within three-hours after hospital arrival and within $12 \mathrm{~h}$ after stroke symptom onset. If time of symptom onset is unclear or patient is awakening with stroke symptoms, the time of onset will be the time that the patient was last known to be normal.

4. Known history of type 2 diabetes mellitus and admission blood glucose $>110 \mathrm{mg} / \mathrm{dL}(>6 \cdot 10 \mathrm{mmol} / \mathrm{L})$ OR admission blood glucose $\geq 150 \mathrm{mg} / \mathrm{dL}$ ( $\geq 8.32 \mathrm{mmol} / \mathrm{L}$ ) in those without known diabetes mellitus.

5. Baseline NIHSS score 3-22.

6. Prestroke modified Rankin Scale score $=0$ (able to walk, look after own affairs, and no residual symptoms from prior stroke).

7. Able to provide a valid informed consent to enroll (self or legally authorized representative).

Exclusion

1. History of type 1 diabetes mellitus.

2. Substantial preexisting neurological or psychiatric illness that could confound the neurological or other outcome assessment.

3. Having received experimental therapy for the enrollment stroke. IV tPA up to $4.5 \mathrm{~h}$ or IA tPA are allowed as are endovascular therapies using FDA cleared devices. Non-FDA cleared devices are considered experimental and are excluded.

4. Known to be currently pregnant or breast-feeding.

5. Unlikely to survive 90 days due to another serious condition.

6. Inability to follow the protocol or return for the 90-day follow-up.

7. Renal dialysis, including haemo- or peritoneal.

FDA, Food and Drug Administration; IA, intra-arterial; IV, intravenous; NIHSS, National Institutes of Health Stroke Scale; tPA, tissue plasminogen activator. 
hyperglycemia that resolves early and spontaneously during hospitalization $(10,13,19)$. Thus, the SHINE eligibility criteria attempt to exclude patients with self-limited hyperglycemia.

Previous data show that patients with the mildest strokes generally have good outcomes and those with the most severe strokes generally have poor outcomes. Therefore, the mildest $($ NIHSS $<3)$ and most severe $($ NIHSS $>22)$ stroke patients are excluded from the SHINE trial to avoid obscuring a treatment difference between the groups. Also excluded are patients for whom clinical equipoise for treatment is absent (e.g., those with type 1 diabetes or pregnancy), those at excessive risk from either intervention (e.g., receiving renal dialysis), those at risk for loss to follow-up (inability to return), and those with confounding that would preclude accurate estimation of treatment effect (other experimental interventions, substantial preexisting neurological or psychiatric disease) (Table 1).

Additionally, as with the sliding dichotomy some patients require a 90 -day mRS of 0 to classify as having favorable outcome, those with residual symptoms from prior stroke or who are unable to live independently due to preexisting disabilities are excluded from this trial.

\section{Randomization}

The SHINE trial utilizes a web-based central randomization system that employs a combination of covariate balance (20) and response adaptive randomization (RAR) (21) methods. The prognostic variables considered at the time of randomization include baseline NIHSS strata (3-7,8-14,15-22), use of IV tPA thrombolysis (yes/no), and clinical center. Randomization is initially $1: 1$, but as the trial progresses this ratio may change based on two factors, the prevention of serious imbalances in the prespecified prognostic variables and the favorable outcome rate in each treatment group. This randomization design is aimed to preserve the randomness of treatment assignment, prevent serious imbalance in important baseline prognostic variables, and promote subject recruitment while preserving the statistical test power.

\section{Treatment}

There are two treatment groups in the SHINE trial (Table 2). One group receives continuous IV insulin infusion to maintain blood glucose of $80-130 \mathrm{mg} / \mathrm{dL}(4 \cdot 44-7 \cdot 21 \mathrm{mmol} / \mathrm{L})$. An FDA cleared computerized decision support tool, GlucoStabilizer ${ }^{\circledR}$ (22) [Alere Informatics Solutions (AIS), Charlottesville, VA, USA], recommends the insulin drip rate to maintain the glucose in the target range. The other group receives only SQ insulin to maintain blood glucose of $80-179 \mathrm{mg} / \mathrm{dL}(4 \cdot 44-9.93 \mathrm{mmol} / \mathrm{L})$ (Table 2). The glucose values are based on point of care capillary glucose testing. All patients are treated with a combination of IV and SQ study medications, some of which are insulin and some are normal saline to maintain the blind (Table 2). All prestroke antidiabetes medications are held throughout the treatment period. All patients are treated in hospital units that support IV insulin infusion. At the completion of the 72-h study treatment period, the treating team determines the subsequent standard care regimen. For patients who are ready for discharge prior to $72 \mathrm{~h}$, the study medications are discontinued at least six-hours prior to discharge. If study treatment must be temporarily interrupted for standard care reasons, procedures for pausing and restarting the study treatment are provided.

The treating nurses enter all glucose levels and insulin treatments in both groups into laptop computers, which are instantly transmitted to an AIS server in Charlottesville, VA, USA. AIS transfers all study data to the Statistical and Data Management Center every $24 \mathrm{~h}$.

Hypoglycemia is defined as blood glucose $<70 \mathrm{mg} / \mathrm{dL}$ $(<3.88 \mathrm{mmol} / \mathrm{L})$ and severe hypoglycemia as $<40 \mathrm{mg} / \mathrm{dL}$ $(<2 \cdot 22 \mathrm{mmol} / \mathrm{L})$, but to prevent hypoglycemia, study medications are held and IV glucose is given in both groups whenever the blood glucose levels fall to $<80 \mathrm{mg} / \mathrm{dL}(<4.44 \mathrm{mmol} / \mathrm{L})$. In the control group, patients receive $25 \mathrm{~mL}$ of $50 \%$ dextrose (D50), glucose level is rechecked every $15 \mathrm{~min}$, and additional D50

Table 2 Outline of subcutaneous and intravenous study treatments in the SHINE protocol

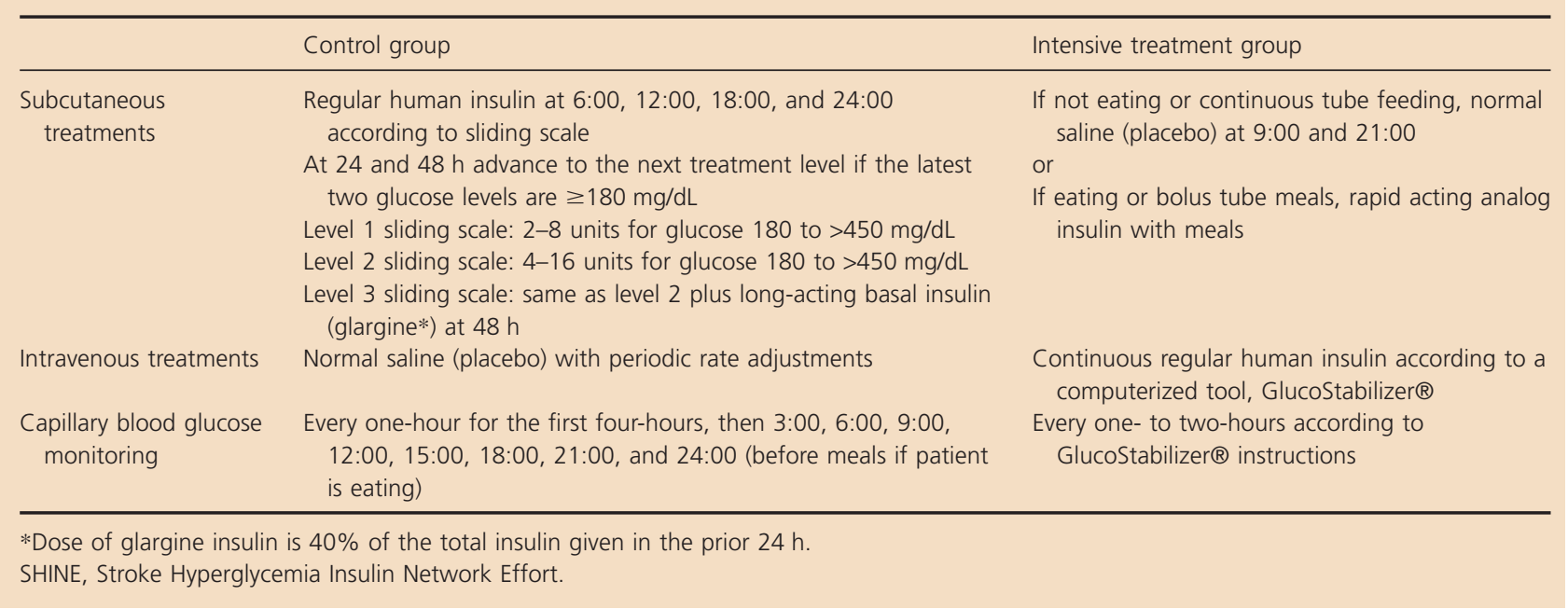


is given after each check until the glucose is $\geq 80 \mathrm{mg} / \mathrm{dL}$ $(\geq 4.44 \mathrm{mmol} / \mathrm{L})$. In the intervention group, the GlucoStabilizer ${ }^{\circledR}$ program recommends a calculated dose of D50 based on the blood glucose level (22). The computer sounds an alarm to recheck the glucose level every $15 \mathrm{~min}$ and recommends additional D50 doses until the glucose is $\geq 80 \mathrm{mg} / \mathrm{dL}$ ( $\geq 4.44 \mathrm{mmol} / \mathrm{L})$.

When blood glucose is $<70 \mathrm{mg} / \mathrm{dL}(<3.88 \mathrm{mmol} / \mathrm{L})$ in either group, a confirmatory blood sample is sent to the laboratory. Additionally, a hypoglycemia symptomatic questionnaire (23) and a neurological examination are done every $15 \mathrm{~min}$ until resolution.

\section{Primary outcome}

The primary efficacy outcome is baseline severity adjusted 90-day mRS assessed using a sliding dichotomy to identify favorable outcomes, also known as responder analysis (16). The responder analysis dichotomizes mRS scores as favorable or unfavorable based on the baseline NIHSS measured at enrollment and the 90-day mRS and was chosen to provide a more sensitive measure of clinical effect. Patients in the lowest baseline severity tertile (NIHSS 3-7) need to have a 90-day mRS score of 0 to achieve favorable outcome. Patients with baseline NIHSS 8-14 can have a 90-day mRS score 0-1 for favorable outcome, and those with baseline NIHSS 15-22 can have a 90-day mRS score 0-2 for favorable outcome, as used in previous trials (17).

\section{Secondary outcomes}

Secondary outcomes include favorable outcome as measured by the NIHSS, Barthel Index, and the Stroke Specific Quality of Life scale (24) at 90 days. Blood glucose control and protocol adherence will also be analyzed.

\section{Data and safety monitoring}

Adverse events are collected during the study treatment period, and serious adverse events are collected throughout the entire study period. The primary safety outcome is the rate of severe hypoglycemia determined as the percentage of patients in each group having at least one blood glucose measurement $<40 \mathrm{mg} / \mathrm{dL}$ $(<2 \cdot 22 \mathrm{mmol} / \mathrm{L})$ during the treatment period.

A study appointed independent safety monitor and an NIHNINDS appointed Data and Safety Monitoring Board (DSMB) oversee safety in the SHINE trial. The DSMB meets every sixmonths to review study progress and accumulated data. Their main responsibilities are to ensure that study participants are not exposed to unnecessary or unreasonable risks and that the study is conducted with high scientific and ethical standards. The DSMB is assisted by an independent safety monitor who reviews and adjudicates all serious adverse events throughout the study. The independent safety monitor also fields safety concerns from the investigators during study treatment.

\section{Sample size}

The sample size estimate was based on data from the two NIH funded pilot trials $(10,11)$, as well as other relevant acute stroke trials $(25,26)$. These data supported an estimate of $25 \%$ favorable outcome rate in the control group. The minimal clinically relevant absolute difference in favorable outcome between the two treatment groups was estimated to be $7 \%$ (control group $=25 \%$; intervention group $=32 \%$ ). The study is therefore powered to detect an absolute 7\% difference in favorable outcome between the groups. The study design includes four interim analyses for both efficacy and futility of the primary outcome (after 500, 700, 900, and 1100 patients complete the study) and a final analysis for a total of five planned analyses of the primary outcome. Including a 3\% nonadherence rate and the four interim analyses, approximately 1400 randomized patients are needed to provide $80 \%$ power with a two-sided type I error rate of 0.05.

In the event that the control group favorable outcome rate is higher than $25 \%$, a blinded sample size reestimation will be done prior to the first interim efficacy/futility analysis using the approach of Gould and Shih (27). The overall favorable outcome rate of the study population will be estimated using the interim data for the sole purpose of sample size reestimation (not for interim testing of a treatment effect).

\section{Statistical analysis}

The primary analysis for the SHINE trial will compare the proportion of subjects in each treatment group with favorable outcome after controlling for baseline NIHSS and standard IV tPA. Outcome differences will be analyzed under the intentionto-treat principle (all randomized participants included) and adjusted relative risks will be reported with two-sided 95\% confidence intervals. Additional analyses will identify potential prognostic variables as covariates for secondary analyses of the primary outcome. Specific variables include age, gender, race ethnicity, race-ethnicity, admission blood glucose, previous stroke, lacunar stroke sub-type, and time from stroke onset to randomization.

The four interim analysis plans described above use the error spending function method with O'Brien and Fleming (OBF)-type stopping guidelines $(28,29)$. The OBF-type boundary is considered conservative as its boundaries make it unlikely to terminate a study early by requiring overwhelming early evidence of efficacy or futility. It spends smaller amounts of alpha at the first look and gradually increases the spending as more information is acquired while maintaining the overall type I and II error rates. For example, under the null hypothesis of no difference between the two treatment groups, there is a $4 \%$ chance of stopping the trial for futility at the first look, $10 \%$ at the second, $25 \%$ at the third, and $35 \%$ at the fourth. Overall, there is approximately a $75 \%$ chance of stopping the trial early for futility if there is no difference. Under the alternative hypothesis, there is an $11 \%$ chance of stopping the trial early for overwhelming efficacy at the first look and a $66 \%$ chance overall of stopping early. These analyses will 
begin once final outcome data are available from roughly onethird of the study population.

\section{Trial organization and funding}

The SHINE trial is funded by the NIH/NINDS. Recognizing the effort and skill needed to successfully run the various aspects of a large acute clinical trial, the SHINE trial has three principal investigators, each with a specified focus. The administrative PI (K. C. J.) is the main contact person for SHINE and chairs the executive committee. The protocol PI (A. B.) oversees the treatment protocols. The recruitment PI (C. E. H.) is focused on patient recruitment and retention. A study endocrinologist (R. J.) oversees the insulin treatments and all relevant metabolic issues. The SHINE trial is conducted primarily in collaboration with the NINDS funded Neurological Emergencies Treatment Trials (NETT) Network, as well as numerous ancillary (non-NETT) sites for patient enrollment. The NETT includes 22 hub and spoke complexes (Appendix) (NETT PI is W. G. B.). The Clinical Coordinating Center for the NETT and therefore SHINE is at the University of Michigan. The Statistical and Data Management Center for the NETT and for SHINE is the Medical University of South Carolina Data Coordinating Unit, directed by the SHINE Statistical PI (V. L. D.). A total of approximately 60 sites are expected to participate in this trial.

\section{Summary}

The SHINE trial is designed to address key questions about the management of hyperglycemia during acute ischemic stroke. This trial has the potential to impact the management of a substantial proportion of acute stroke patients. This trial design is scientifically rigorous, including RAR and baseline severity adjusted double-blind primary outcome assessment. Continuous intravenous insulin infusion based on a computerized decision support tool will be compared with a standard SQ insulin sliding scale. Recruitment started in April 2012 and is expected to be completed in 2016.

\section{Acknowledgements}

The SHINE trial is funded by an NIH/NINDS grant U01NS069498 and registered at ClinicalTrials.gov, \#NCT01369069. The NETT Clinical Coordinating Center is funded by the NIH/NINDS grant U01-NS056975. The NETT Statistical and Data Management Center is funded by the NIH/NINDS grant U01-NS059041.

\section{Addendum}

After enrolling 57 patients the SHINE DSMB approved a protocol modification allowing enrolment of patients with a pre-stroke mRS of 0 or 1 if their baseline NIHSS is $8-22$. However, patients with baseline NIHSS 3-7 must have a pre-stroke mRS of 0 to permit reaching a potential favorable outcome that is based on a sliding dichotomy of the mRS according to the baseline NIHSS score.

\section{References}

1 Gentile NT, Seftchick MW, Huynh T, Kruus LK, Gaughan J. Decreased mortality by normalizing blood glucose after acute ischemic stroke. Acad Emerg Med 2006; 13:174-80.

2 Williams LS, Rotich J, Qi R et al. Effects of admission hyperglycemia on mortality and costs in acute ischemic stroke. Neurology 2002; 59:67-71.

3 Kruyt ND, Biessels GJ, Devries JH, Roos YB. Hyperglycemia in acute ischemic stroke: pathophysiology and clinical management. Nat Rev Neurol 2010; 6:145-55.

4 de Courten-Myers GM, Kleinholz M, Wagner KR, Myers RE. Normoglycemia (not hypoglycemia) optimizes outcome from middle cerebral artery occlusion. J Cereb Blood Flow Metab 1994; 14:227-36.

5 Meden P, Andersen M, Overgaard K, Rasmussen RS, Boysen G. The effects of early insulin treatment combined with thrombolysis in rat embolic stroke. Neurol Res 2002; 24:399-404.

6 Zhu CZ, Auer RN. Optimal blood glucose levels while using insulin to minimize the size of infarction in focal cerebral ischemia. J Neurosurg 2004; 101:664-8.

7 Ntaios G, Egli M, Faouzi M, Michel P. J-shaped association between serum glucose and functional outcome in acute ischemic stroke. Stroke 2010; 41:2366-70.

8 Malmberg K, Ryden L, Efendic S et al. Randomized trial of insulinglucose infusion followed by subcutaneous insulin treatment in diabetic patients with acute myocardial infarction (DIGAMI study): effects on mortality at 1 year. J Am Coll Cardiol 1995; 26:57-65.

9 Van den Berghe G, Wilmer A, Hermans G et al. Intensive insulin therapy in the medical ICU. N Engl J Med 2006; 354:449-61.

10 Bruno A, Kent TA, Coull BM et al. Treatment of hyperglycemia in ischemic stroke (THIS): a randomized pilot trial. Stroke 2008; 39:384-9.

11 Johnston KC, Hall CE, Kissela BM, Bleck TP, Conaway MR. Glucose regulation in acute stroke patients (GRASP) trial: a randomized pilot trial. Stroke 2009; 40:3804-9.

12 Adams HP Jr, del Zoppo G, Alberts MJ et al. Guidelines for the early management of adults with ischemic stroke: a guideline from the American Heart Association/American Stroke Association Stroke Council, Clinical Cardiology Council, Cardiovascular Radiology and Intervention Council, and the Atherosclerotic Peripheral Vascular Disease and Quality of Care Outcomes in Research Interdisciplinary Working Groups: the American Academy of Neurology affirms the value of this guideline as an educational tool for neurologists. Stroke 2007; 38:1655-711.

13 Gray CS, Hildreth AJ, Sandercock PA et al. Glucose-potassium-insulin infusions in the management of post-stroke hyperglycaemia: the UK glucose insulin in stroke trial (GIST-UK). Lancet Neurol 2007; 6:397406.

14 Kreisel SH, Berschin UM, Hammes HP et al. Pragmatic management of hyperglycaemia in acute ischaemic stroke: safety and feasibility of intensive intravenous insulin treatment. Cerebrovasc Dis 2009; 27:16775 .

15 Walters MR, Weir CJ, Lees KR. A randomised, controlled pilot study to investigate the potential benefit of intervention with insulin in hyperglycaemic acute ischaemic stroke patients. Cerebrovasc Dis 2006; 22:116-22.

16 Adams HP, Jr, Leclerc JR, Bluhmki E, Clarke W, Hansen MD, Hacke W. Measuring outcomes as a function of baseline severity of ischemic stroke. Cerebrovasc Dis 2004; 18:124-9.

17 Adams HP, Jr, Effron MB, Torner J et al. Emergency administration of abciximab for treatment of patients with acute ischemic stroke: results of an international phase III trial: abciximab in emergency treatment of stroke trial (AbESTT-II). Stroke 2008; 39:87-99. 
18 Bruno A, Waddell MJ, Potter DJ et al. Opportunity to lower hyperglycaemia faster in patients with acute ischaemic stroke and diabetes. Int J Stroke 2010; 5:338-9.

19 Wong AA, Schluter PJ, Henderson RD, O'Sullivan JD, Read SJ. Natural history of blood glucose within the first 48 hours after ischemic stroke. Neurology 2008; 70:1036-41.

20 Zhao W, Hill MD, Palesch Y. Minimal sufficient balance - a new strategy to balance baseline covariates and preserve randomness of treatment allocation. Stat Methods Med Res 2012, doi: 10.1177/ 0962280212436447.

$21 \mathrm{Hu}$ F, Rosenberger WF. The Theory of Response Adaptive Randomization in Clinical Trials. New York, Wiley, 2006.

22 Juneja R, Roudebush C, Kumar N et al. Utilization of a computerized intravenous insulin infusion program to control blood glucose in the intensive care unit. Diabetes Technol Ther 2007; 9:232-40.

23 Towler DA, Havlin CE, Craft S, Cryer P. Mechanism of awareness of hypoglycemia. Perception of neurogenic (predominantly cholinergic) rather than neuroglycopenic symptoms. Diabetes 1993; 42:1791-8.

24 Williams LS, Weinberger M, Harris LE, Biller J. Measuring quality of life in a way that is meaningful to stroke patients. Neurology 1999; 53:1839-43.

25 The National Institute of Neurological Disorders and Stroke rt-PA Stroke Study Group. Tissue plasminogen activator for acute ischemic stroke. N Engl J Med 1995; 333:1581-7.
26 Palesch YY, Hill MD, Ryckborst KJ, Tamariz D, Ginsberg MD. The ALIAS pilot trial: a dose-escalation and safety study of albumin therapy for acute ischemic stroke - II: neurologic outcome and efficacy analysis. Stroke 2006; 37:2107-14.

27 Gould AL, Shih WJ. Modifying the design of ongoing trials without unblinding. Stat Med 1998; 17:89-100.

28 O'Brien PC, Fleming TR. A multiple testing procedure for clinical trials. Biometrics 1979; 35:549-56.

29 Lan KKG, Demets DL. Discrete sequential boundaries for clinical trials. Biometrika 1983; 70:659-63.

\section{Supporting information}

Additional Supporting Information may be found in the online version of this article at the publisher's web-site:

Appendix S1. NETT hub sites, Ancillary sites and SHINE Executive Committee 